\title{
Correction to: Age-related changes to environmental exposure: variation in the frequency that young children place hands and objects in their mouths
}

\author{
Laura H. Kwong $^{1} \cdot$ Ayse Ercumen $^{2,3} \cdot$ Amy J. Pickering $^{4} \cdot$ Leanne Unicomb $^{5} \cdot$ Jennifer Davis $^{1,6} \cdot$ Stephen P. Luby $^{6}$
}

Published online: 27 August 2019

(c) The Author(s), under exclusive licence to Springer Nature America, Inc. 2019

Correction to: Official journal of the International Society of Exposure Science https://doi.org/10.1038/s41370-019-0115-8

In the original version of this article, locationindependent hand-mouthing frequencies, object-mouthing frequencies, and frequencies of hand contact with soil were incorrectly reported. Mobility was also incorrectly stated as not associated with location-independent object-mouthing. (The location-specific frequencies reported in the original article were correct.) We have accordingly corrected the manuscript text, Table 2, Figures 1, 3, and 4, and the supplemental information in the pdf and html versions of the article.
Laura H. Kwong

lakwong@stanford.edu

1 Department of Civil and Environmental Engineering, Stanford University, Stanford, CA, USA

2 Department of Forestry and Environmental Resources, North Carolina State University, Raleigh, NC, USA

3 Division of Epidemiology and Biostatistics, University of California, Berkeley, CA, USA

4 Department of Civil and Environmental Engineering, Tufts University, Medford, MA, USA

5 International Centre for Diarrhoeal Disease Research, Bangladesh, Dhaka, Bangladesh

6 Woods Institute for the Environment, Stanford University, Stanford, CA, USA 
Table 2 Prevalence and frequency of observed soil (top) and feces (bottom) ingestion during observations of young children

\begin{tabular}{|c|c|c|c|c|c|c|c|}
\hline \multirow[t]{2}{*}{ Age group } & \multirow[t]{2}{*}{ Study } & \multirow{2}{*}{$\begin{array}{l}\text { Children } \\
\text { observed placing } \\
\text { soil in mouth }\end{array}$} & \multirow{2}{*}{$\begin{array}{l}\text { Total children } \\
\text { observed }\end{array}$} & \multirow[t]{2}{*}{$\%$} & \multicolumn{3}{|c|}{ Frequency (times/h) } \\
\hline & & & & & median & mean & $\mathrm{sd}$ \\
\hline \multirow[t]{4}{*}{$3-5$ months } & This study & 0 & 2 & 0 & NA & NA & NA \\
\hline & Bauza, 2018 ${ }^{\mathrm{a}}$ & 0 & 55 & - & - & - & - \\
\hline & Kwong, 2016 & 2 & 21 & 10 & 0.0 & 0.1 & 0.2 \\
\hline & Ngure, 2013 & 1 & 7 & 14 & 1.7 & 1.7 & NA \\
\hline \multirow[t]{5}{*}{ 6-11 months } & This study & 8 & 19 & 42 & 1.1 & 1.3 & 1.0 \\
\hline & Bauza, 2018 ${ }^{\mathrm{a}}$ & 13 & 49 & 27 & - & - & - \\
\hline & Kwong, 2016 & 29 & 104 & 28 & 0.0 & 0.3 & 0.0 \\
\hline & George, 2015 & 11 & 32 & 34 & - & - & - \\
\hline & Ngure, 2013 & 0 & 7 & 0 & - & NA & - \\
\hline \multirow[t]{5}{*}{$12-23$ months } & This study & 8 & 31 & 26 & 0.6 & 0.9 & 0.8 \\
\hline & Bauza, 2018 ${ }^{\mathrm{a}}$ & 37 & 98 & 38 & - & - & - \\
\hline & Kwong, 2016 & 8 & 23 & 35 & 2.5 & 1.2 & 2.5 \\
\hline & George, 2015 & 25 & 142 & 18 & - & - & - \\
\hline & Ngure, 2013 & 2 & 7 & 29 & - & 2.0 & - \\
\hline \multirow[t]{3}{*}{ 24-35 months } & This study & 2 & 26 & 8 & 0.4 & 0.4 & 0.3 \\
\hline & Bauza, $2018^{\mathrm{a}}$ & 19 & 100 & 3 & - & - & - \\
\hline & George, 2015 & 2 & 42 & 5 & - & - & - \\
\hline \multirow[t]{2}{*}{ 36-47 months } & This study & 0 & 6 & 0 & NA & NA & NA \\
\hline & Bauza, 2018 & 3 & 120 & 3 & - & - & - \\
\hline $3-23$ months & Reid, 2018 & 14 & 30 & 47 & - & - & - \\
\hline \multirow[t]{3}{*}{$3-5$ months } & This study & 0 & 2 & 0 & NA & NA & NA \\
\hline & Kwong, 2016 & 0 & 21 & 0 & - & NA & NA \\
\hline & Ngure, 2013 & 0 & 7 & 0 & - & NA & - \\
\hline \multirow[t]{3}{*}{ 6-11 months } & This study & 1 & 19 & 5 & 0.6 & 0.6 & NA \\
\hline & Kwong, 2016 & 3 & 104 & 3 & 0.0 & 0.0 & 0.0 \\
\hline & Ngure, 2013 & 1 & 7 & 14 & - & 0.2 & - \\
\hline \multirow[t]{3}{*}{$12-23$ months } & This study & 0 & 31 & 0 & NA & NA & NA \\
\hline & Kwong, 2016 & 1 & 23 & 4 & 0.0 & 0.0 & 0.0 \\
\hline & Ngure, 2013 & 3 & 7 & 43 & - & 0.5 & - \\
\hline 24-35 months & This study & 0 & 27 & 0 & NA & NA & NA \\
\hline 36-47 months & This study & 0 & 6 & 0 & NA & NA & NA \\
\hline $3-23$ months & Reid, 2018 & 1 & 30 & 3 & NA & NA & NA \\
\hline 6-30 months & George, 2015 & 1 & 216 & 0 & & $>0.2$ times $/ \mathrm{h}$ & \\
\hline 0-59 months & Marquis, $1990^{\mathrm{b}}$ & Not given & 21 & - & - & 0.3 & 0.4 \\
\hline
\end{tabular}

${ }^{a}$ Caregiver-reported ingestion events in the past 7 days, rather than observed events

${ }^{\mathrm{b}}$ Mouthing fecally contaminated hands and objects

- Value not provided 

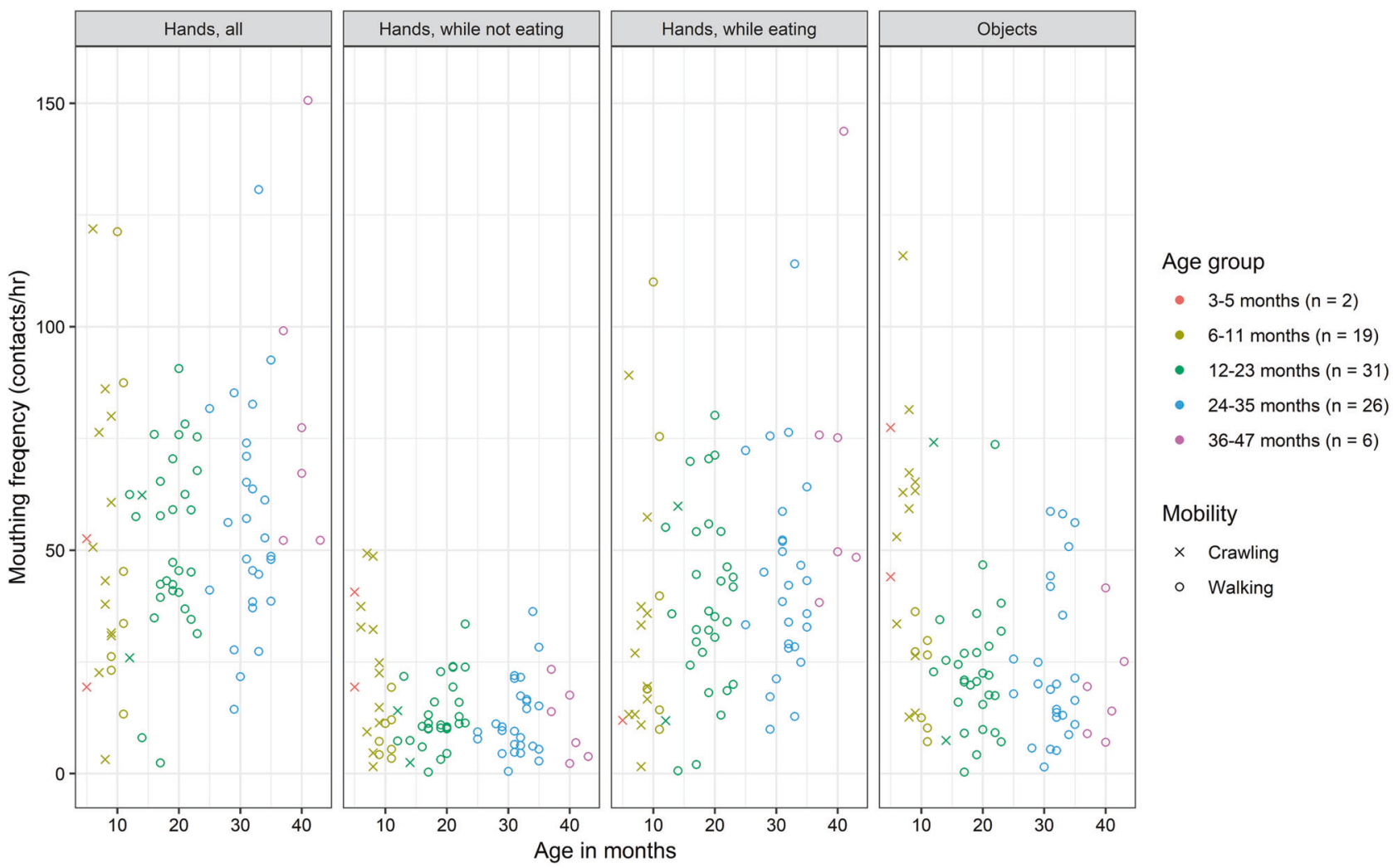

Fig. 1 Frequency of hand-to-mouth contacts not related to eating, hand-to-mouth contacts related to eating, all hand-to-mouth contacts, and objectto-mouth contacts among young children in rural Bangladesh

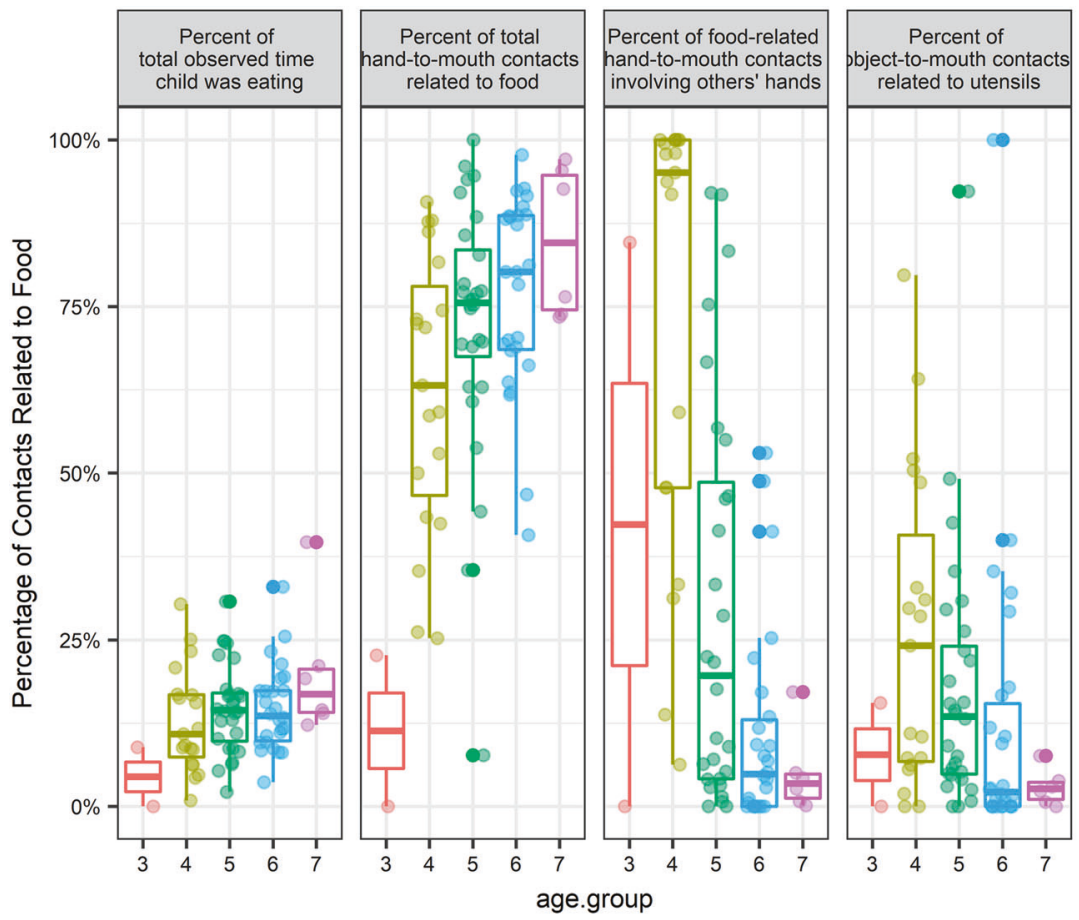

Age group

3-5 months $(n=2)$

6-11 months $(n=19)$

12-23 months $(n=31)$

24-35 months $(n=26)$

36-47 months $(n=6)$

$\times \quad$ Crawling

$\circ$ Walking

Fig. 3 Percentage of hand-to-mouth contacts that occurred during eating or involved other individuals and the percentage of object-to-mouth contacts that involved utensils among young children in rural Bangladesh. The dark middle lines denote the median, the edges of the boxes mark the 75 th and 25 th percentile, and the whiskers extend to values \pm 1.5 times the interquartile range 

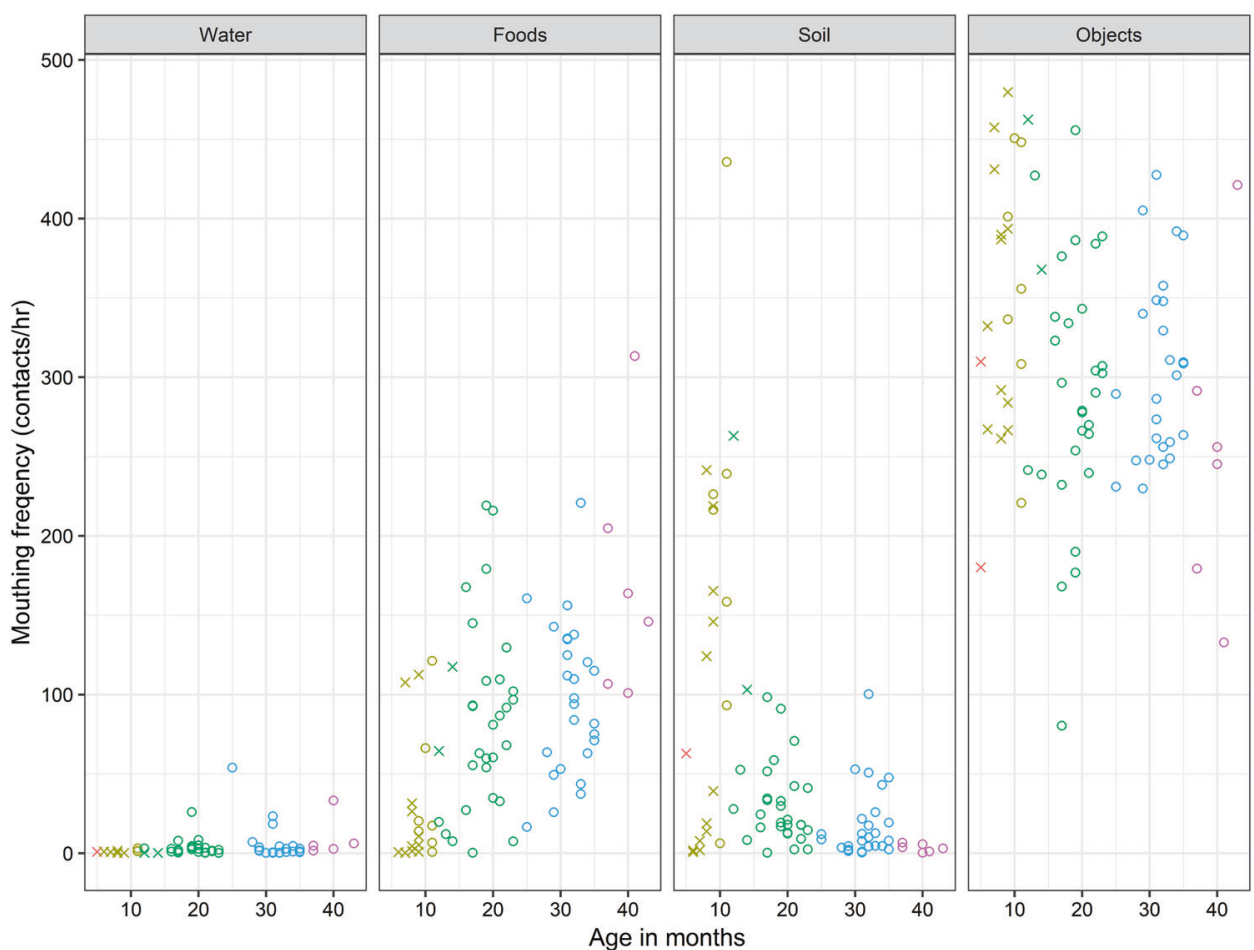

Age group

- 3-5 months ( $n=2)$

- 6-11 months $(n=19)$

- $12-23$ months $(n=31$ )

- 24-35 months $(n=26)$

- 36-47 months $(n=6)$

Mobility

$\times$ Crawling

- Walking

Fig. 4 Frequency of touching water, foods, soil, and objects among young children in rural Bangladesh 